\title{
Similarity Measures of Tangent, Cotangent and Cosines in Neutrosophic Environment and their Application in Selection of Academic Programs
}

\author{
Muhammad Naveed \\ Jafar \\ Lahore Garrison \\ University 54000 \\ Lahore, Pakistan
}

\author{
Asma Farooq \\ Lahore Garrison \\ University 54000 \\ Lahore, Pakistan
}

\author{
Komal Javed \\ Lahore Garrison \\ University 54000 \\ Lahore, Pakistan
}

\author{
Nazia Nawaz \\ Lahore Garrison \\ University 54000 \\ Lahore, Pakistan
}

\begin{abstract}
Similarity measures have wide range of applications in realworld such as patterns, face recognitions, codding etc. In this paper it is intended to determine the tangent, cosine and cotangent similarity measure for single valued Neutrosophic sets and will compare the accuracy of all above similarity measures and applied it in decision making problems such as selection of an academic programs.
\end{abstract}

\section{Keywords}

Similarity Measures, Neutrosophic Sets, Tangent Measures, Cosine Measures, Cotangent Measures

\section{INTRODUCTION}

In daily life, everyone deals with uncertainty. If anyone toss a coin one will get either a head or tail but are not sure about outcomes so to tackle with issues like uncertainty and to make decisions in practical applications many theories have been proposed and some of them are as follows: The concept of Probability theory was first introduced by Blaise Pascal (1632-62) and Pierre Fermet (1601-65). Then in 1965, the theory of fuzzy sets (FSs) was offered by Zadeh [48]. Also the notion of IVFS was suggested by Zadeh [49]. In 1986, the perception of IFS was offered by Atanassov [2]. Atanassov and Gorgov [3] gave the perception of IVIFSs in 1989 and in 1998 the concept of NS was suggested by Smarandache [34]. By his definition, the NS is specified by truthiness, indeterminacy and falseness and for analytical applications its value can be taken from usual and unusual subsets of ]0, 1[. an idea of SVNSs was presented by Wang et al [40] by recognizing the difficulty in application of NSs in realistic complications. To describe inadequate, unpredictable, uncertain and inaccurate data, it suggest us additional prospect that evident the world.

In different fields such as educational problem [21,22] , image processing $[8,11,50]$, medical diagnosis $[1,19,45,46]$, social problems [23,26], conflict resolution [27] etc have been applied and studied in NSs and its numerous extensions. In all scientific fields, the concept of similarity is very important. To calculate similarity measure between FSs many methods have been proposed that was given by Wang [39] and studied by Hyung et al [12], Chen et al [9], Pappis and Karacapildis [25], Chen [10]. Concerning indeterminacy these techniques are not proficient of dealing with similarity measure. Similarity measures for SVNS and Neutrosophic sets [4, 5, 6, $20,40,41$, and 42] have lectured in literature.

In 2013 Hausdrof distance extended to NSs by Broumi and Smarandache [5] that plays a vital role in real-world application, computer assisted surgery, particularly in numerous pictorial tasks etc. The correlation coefficient between interval Neutrosophic sets were offered by Broumi and Smarandache in 2013. Numerous similarity measures of SVNSs depend on membership grades, distance, entropy measure for a SVNS and a matching function were studied by Majumdar and Smanta [20]. For SVNSs, the Similarity measure based on distance and its application in DM problem were proposed by Ye [40] in 2013. An example of SVNS and interval valued Neutrosophic set, three vector similarity measure for SNSs were proposed by Ye [43] including the Dice, cosine and Jaccard similarity and with basic Neutrosophic data applied it to MCDM problems.

The SM based on tangent function of IFSs and its application in medical analysis was suggested by Mondal and Paramanik [24].By using Euclidean and Hamming distance, the SM of INS was explained by Jun [42] and also gave an instance in DM problem. The SM based on cotangent function between Intuitionistic fuzzy sets and its application in medical analysis was introduced by Tian [37].Cosine SM of IVNSs was suggested by Samarandache and Broumi [7]. However due to limitation in prevailing Cosine SM of SNSs in vector space. An improved cosine SM of SNSs which is based on Cosine function and its application in medical analysis was proposed by Ye [44]. Jafar et al $(2019,2020)$ use fuzzy, Intuitionistic and Neutrosophic logics in different decision making problems in [13-18]. Saqlain et al worked on different decision making problems using Neutrosophic theory. Researchers doing a fantastic job in MCDM problems using these uncertain conditions in [28-33]

This paper is about Neutrosophic introduction (section-2), Tangent similarity measure for SVNSs (section-3), Procedure of decision making (section-4), Cosine similarity measure for SVNSs (section-5), Cotangent SM for SVNSs (section-6), Applications named as selection of an academic program and identification of diseases by using tangent, cosine and cotangent SM (section-7).

\section{PRELIMINARIES}

i. Neutrosophic Sets $[34,35]$

Suppose there be a universal setU. Further a NS $\mathcal{M}$ is expressed as:

$\mathcal{M}=\left\{<\breve{z}: \mathbb{T}_{\mathcal{M}}(\check{z}), \mathbb{I}_{\mathcal{M}}(\check{z}), \mathbb{F}_{\mathcal{M}}(\check{z})>, \check{z} \in \mathbb{U}\right\}$, Thus

$\mathbb{T}, \mathbb{I}, \mathbb{F}: \mathbb{U} \rightarrow]^{-\cdot} 0,1^{+\cdot}[$ represent truthiness, indeterminacy and falseness correspondingly of the point $\check{z} \in \mathbb{U}$ via set $\mathcal{M}$ fulfill the resulting limitation:

$$
-\dot{0} \leq \sup \mathbb{T}_{\mathcal{M}}(\check{\mathrm{z}})+\sup \mathbb{I}_{\mathcal{M}}(\check{\mathrm{z}})+\sup \mathbb{F}_{\mathcal{M}}(\check{\mathrm{z}}) \leq \dot{3}^{+}
$$


For analytical application, the values of truthiness, indeterminacy and falseness can be taken by subsets of ]$^{-} \dot{0}, \dot{1}^{+}$[ can be taken as the NS value. For practical usage, we will take $[\dot{0}, \dot{1}]$ rather than $]^{-} \dot{0}, \dot{1}^{+}$as the application of $]-\dot{0}, \dot{1}^{+}[$is a challenging task in mathematical complications. Now let two NSs,

$\left.\mathcal{M}_{N S}=\left\{<\check{z}: \mathbb{T}_{\mathcal{M}}(\check{z}), \mathbb{I}_{\mathcal{M}}(\check{z}), \mathbb{F}_{\mathcal{M}}(\check{z})\right\rangle, \check{z} \in \check{X}\right\}$,

$\mathcal{N}_{N S}=\left\{<\check{z}: \mathbb{T}_{\mathcal{N}}(\check{z}), \mathbb{I}_{\mathcal{N}}(\check{z}), \mathbb{F}_{\mathcal{N}}(\check{z})>\check{z} \in \check{X}\right\}$, Following are the relation of above NSs:

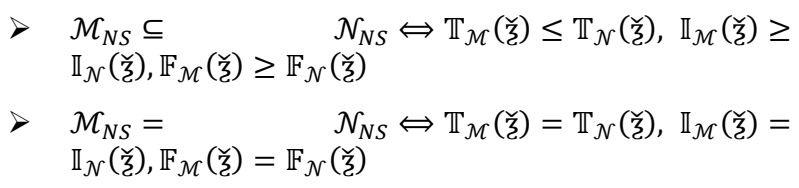

\section{ii. SINGLE VALUED NEUTROSOPHIC SETS [38]}

Suppose there be an universe of points $\breve{X}$ along general point in $\check{X}$ symbolized by $\breve{z}$. A single valued neutrosophic set $\mathcal{M}$ in $\check{X}$ is categorized via a function of truthiness $\mathbb{T}_{\mathcal{M}}(\check{Z})$, indeterminacy $\mathbb{I}_{\mathcal{M}}(\breve{z})$, and falseness $\mathbb{F}_{\mathcal{M}}(\breve{z})$ for every $\breve{z}$ in $\check{X}$, $\mathbb{T}_{\mathcal{M}}(\breve{z}), \mathbb{I}_{\mathcal{M}}(\breve{z}),, \mathbb{F}_{\mathcal{M}}(\breve{z}) \in[\dot{0}, \dot{1}]$. If $\check{X}$ is continual, then the single valued Neutrosophic set $\mathcal{M}$ will be:

$$
\mathcal{M}=\int_{\check{X}} \frac{\left\langle\mathbb{T}_{\mathcal{M}}(\breve{z}), \mathbb{I}_{\mathcal{M}}(\breve{z}), \mathbb{F}_{\mathcal{M}}(\breve{z})>\right.}{\check{z}}: \check{z} \in \check{X}
$$

Now if $\check{X}$ is discrete, then single valued Neutrosophic set $\mathcal{M}$ can be presented as:

$$
\mathcal{M}=\sum_{i=1}^{\dot{n}} \frac{<\mathbb{T}_{\mathcal{M}}\left(\check{\mathrm{z}}_{i}\right), \mathbb{I}_{\mathcal{M}}\left(\check{\mathrm{z}}_{i}\right), \mathbb{F}_{\mathcal{M}}\left(\check{\mathrm{z}}_{i}\right)>}{\check{\mathrm{z}}_{i}}: \check{\check{z}}_{i} \in \check{X}
$$

Now let two single valued Neutrosophic sets,

$$
\begin{aligned}
& \left.\mathcal{M}_{\text {SVNS }}=\left\{<\check{z}: \mathbb{T}_{\mathcal{M}}(\check{z}), \mathbb{I}_{\mathcal{M}}(\check{z}), \mathbb{F}_{\mathcal{M}}(\check{z})\right\rangle, \check{z} \in \check{X}\right\} \text { and } \\
& \left.\mathcal{N}_{\text {SVNS }}=\left\{<\check{z}: \mathbb{T}_{\mathcal{N}}(\check{z}), \mathbb{I}_{\mathcal{N}}(\check{z}), \mathbb{F}_{\mathcal{N}}(\check{z})\right\rangle, \check{z} \in \check{X}\right\},
\end{aligned}
$$

Following are the relations of above SVNSs

$$
\begin{aligned}
& >\quad \mathcal{M}_{S V N S} \subseteq \mathcal{N}_{S V N S} \Leftrightarrow \mathbb{T}_{\mathcal{M}}(\check{\mathrm{z}}) \leq \mathbb{T}_{\mathcal{N}}(\check{\mathrm{z}}), \\
& \mathbb{I}_{\mathcal{M}}(\check{z}) \geq \mathbb{I}_{\mathcal{N}}(\check{z}), \mathbb{F}_{\mathcal{M}}(\check{z}) \geq \mathbb{F}_{\mathcal{N}}(\check{z}) \\
& >\quad \mathcal{M}_{S V N S}=\mathcal{N}_{S V N S} \Leftrightarrow \mathbb{T}_{\mathcal{M}}(\check{\xi})=\mathbb{T}_{\mathcal{N}}(\check{z}), \mathbb{I}_{\mathcal{M}}(\check{\xi})= \\
& \mathbb{I}_{\mathcal{N}}(\breve{z}), \mathbb{F}_{\mathcal{M}}(\breve{z})=\mathbb{F}_{\mathcal{N}}(\breve{z}) \text { for any } \breve{z} \in \check{X}
\end{aligned}
$$

iii. TANGENT SIMILARITY MEASURES FOR SVNSS Suppose there exist two SVNNs,

$\mathcal{M}=<\check{z}:\left(\mathbb{T}_{\mathcal{M}}(\check{z}), \mathbb{I}_{\mathcal{M}}(\check{z}), \mathbb{F}_{\mathcal{M}}(\check{z})\right)>\quad$ and $\quad \mathcal{N}=<$ $\check{z}:\left(\mathbb{T}_{\mathcal{N}}(\check{z}), \mathbb{I}_{\mathcal{N}}(\check{z}), \mathbb{F}_{\mathcal{N}}(\check{z})\right)>$

Similarity among two points is calculated by TS function that depend on direction only, disregarding an effect of space among them is represented as:-

$$
\begin{aligned}
& \mathrm{F}_{S V N S}(\mathcal{M}, \mathcal{N})= \\
& \frac{\dot{1}}{\dot{n}} \sum_{i=i}\{\dot{1}- \\
& \left.\tan \left[\frac{\pi\left[\left|\mathbb{T}_{\mathcal{M}}\left(\check{z}_{i}\right)-\mathbb{T}_{\mathcal{N}}\left(\breve{z}_{i}\right)\right|+\left|\mathbb{I}_{\mathcal{M}}\left(\check{z}_{i}\right)-\mathbb{I}_{\mathcal{N}}\left(\breve{z}_{i}\right)\right|+\left|\mathbb{F}_{\mathcal{M}}\left(\breve{z}_{i}\right)-\mathbb{F}_{\mathcal{N}}\left(\breve{z}_{i}\right)\right|\right]}{12}\right]\right\}
\end{aligned}
$$

Proposition 3.1. The definite Tangent SM $\mathrm{T}_{S V N S}(\mathcal{M}, \mathcal{N})$ between SVNSs $\mathcal{M}$ and $\mathcal{N}$ fulfill the resulting properties
I. $\quad \dot{0} \leq \mathrm{F}_{S V N S}(\mathcal{M}, \mathcal{N}) \leq \dot{1}$
II. $\quad \mathrm{T}_{S V N S}(\mathcal{M}, \mathcal{N})$ if and only if $\mathcal{M}=\mathcal{N}$
III. $\quad \mathrm{F}_{S V N S}(\mathcal{M}, \mathcal{N})=\mathrm{F}_{S V N S}(\mathcal{N}, \mathcal{M})$

IV. If $\mathcal{O}$ is the single valued Neutrosophic set in $\check{X}$ and

$\mathcal{M} \subset \mathcal{N} \subset \mathcal{O}$ as a result

$\mathrm{F}_{S V N S}(\mathcal{M}, \mathcal{O}) \leq \mathrm{F}_{S V N S}(\mathcal{M}, \mathcal{N})$ and $\mathrm{F}_{S V N S}(\mathcal{N}, \mathcal{O})$

$\mathrm{F}_{S V N S}(\mathcal{M}, \mathcal{O}) \leq$

Proofs:

I. The TF's value and the truthiness, indeterminacy and falseness of single valued Neutrosophic sets are in $[\dot{0}, \dot{1}]$, the tangent similarity measure is in between $[\dot{0}, \dot{1}]$.

Therefore

$$
\dot{0} \leq \mathrm{F}_{S V N S}(\mathcal{M}, \mathcal{N}) \leq \dot{1}
$$

\begin{tabular}{|c|c|c|c|c|}
\hline & $\check{\mathcal{C}}_{1}$ & $\check{\mathfrak{C}}_{2}$ & $\ldots$ & $\check{\mathcal{C}}_{\dot{n}}$ \\
\hline$\check{\mathcal{A}}_{1}$ & $\alpha_{11}$ & $\alpha_{12}$ & $\ldots$ & $\alpha_{1 \dot{n}}$ \\
\hline$\check{\mathcal{A}}_{1}$ & $\alpha_{21}$ & $\alpha_{22}$ & $\ldots$ & $\alpha_{2 \dot{n}}$ \\
\hline$\ldots$ & $\ldots$ & $\ldots$ & $\ldots$ & $\ldots$ \\
\hline$\check{\mathcal{A}}_{\dot{m}}$ & $\alpha_{\dot{m} 1}$ & $\alpha_{\dot{m} 2}$ & $\ldots$ & $\alpha_{\dot{m} \dot{n}}$ \\
\hline
\end{tabular}

II. Consider two single valued Neutrosophic sets $\boldsymbol{M}$ and $\mathcal{N}$ and let $\mathcal{M}=\mathcal{N}$ then,

$$
\begin{gathered}
\mathbb{T}_{\mathcal{M}}(\check{\mathrm{z}})=\mathbb{T}_{\mathcal{N}}(\check{\mathrm{z}}), \mathbb{I}_{\mathcal{M}}(\check{\mathrm{z}})=\mathbb{I}_{\mathcal{N}}(\check{\mathrm{z}}) \\
, \mathbb{F}_{\mathcal{M}}(\check{\mathrm{z}})=\mathbb{F}_{\mathcal{N}}(\check{\mathrm{z}})
\end{gathered}
$$

Therefore

Thus $\mathrm{F}_{S V N S}(\mathcal{M}, \mathcal{N})=\dot{\mathbf{1}}$

$$
\begin{array}{r}
\left|\mathbb{T}_{\mathcal{M}}(\check{z})-\mathbb{T}_{\mathcal{N}}(\check{z})\right|=\dot{0} \\
,\left|\mathbb{I}_{\mathcal{M}}(\check{z})-\mathbb{I}_{\mathcal{N}}(\check{z})\right|=\dot{0} \\
,\left|\mathbb{F}_{\mathcal{M}}(\check{z})-\mathbb{F}_{\mathcal{N}}(\check{z})\right|=\dot{\mathbf{0}}
\end{array}
$$

Contrarily,

$$
\begin{aligned}
& \text { If } \mathrm{T}_{\boldsymbol{S V N S}}(\mathcal{M}, \mathcal{N})=\dot{\mathbf{1}} \quad \text { then } \quad \mid \mathbb{T}_{\mathcal{M}}(\check{\mathrm{z}})- \\
& \mathbb{T}_{\mathcal{N}}(\check{z}) \mid=\dot{0} \\
& ,\left|\mathbb{I}_{\mathcal{M}}(\check{\mathrm{z}})-\mathbb{I}_{\mathcal{N}}(\breve{\mathrm{z}})\right|=\dot{\mathbf{0}} \\
& ,\left|\mathbb{F}_{\mathcal{M}}(\check{z})-\mathbb{F}_{\mathcal{N}}(\check{z})\right|=\dot{\mathbf{0}} \text { As } \tan \dot{\mathbf{0}}=\dot{\mathbf{0}}
\end{aligned}
$$

it can be written as

$$
\mathbb{T}_{\mathcal{M}}(\check{\mathrm{z}})=\mathbb{T}_{\mathcal{N}}(\check{\mathrm{z}}) \text {, }
$$

$$
\begin{aligned}
& \mathbb{I}_{\mathcal{M}}(\check{z})=\mathbb{I}_{\mathcal{N}}(\check{z}), \\
& \mathbb{F}_{\mathcal{M}}(\check{z})=\mathbb{F}_{\mathcal{N}}(\check{z})
\end{aligned}
$$

Therefore, $\mathcal{M}=\mathcal{N}$

III. Evidence is clear.

IV. If $\mathcal{M} \subset \mathcal{N} \subset \mathcal{O}$ then

$$
\begin{aligned}
& \mathbb{T}_{\mathcal{M}}(\check{z}) \leq \mathbb{T}_{\mathcal{N}}(\check{z}) \leq \mathbb{T}_{\mathcal{O}}(\check{z}), \\
& \mathbb{I}_{\mathcal{M}}(\check{z}) \geq \mathbb{I}_{\mathcal{N}}(\check{z}) \geq \mathbb{I}_{\mathcal{O}}(\check{z}), \\
& \mathbb{F}_{\mathcal{M}}(\check{\zeta}) \geq \mathbb{F}_{\mathcal{N}}(\check{z}) \geq \mathbb{F}_{\mathcal{O}}(\check{z})
\end{aligned}
$$

Now we have the given inequalities:

$\left|\mathbb{T}_{\mathcal{M}}(\check{\zeta})-\mathbb{T}_{\mathcal{O}}(\check{z})\right| \geq\left|\mathbb{T}_{\mathcal{M}}(\check{z})-\mathbb{T}_{\mathcal{N}}(\check{z})\right|$, 


$$
\begin{aligned}
& \left|\mathbb{T}_{\mathcal{M}}(\check{\mathrm{z}})-\mathbb{T}_{\mathcal{O}}(\check{\mathrm{z}})\right| \geq\left|\mathbb{T}_{\mathcal{N}}(\check{\mathrm{z}})-\mathbb{T}_{\mathcal{O}}(\check{\mathrm{z}})\right| ; \\
& \left|\mathbb{I}_{\mathcal{M}}(\check{\mathrm{z}})-\mathbb{I}_{\mathcal{O}}(\check{\mathrm{z}})\right| \geq\left|\mathbb{I}_{\mathcal{M}}(\check{\mathrm{z}})-\mathbb{I}_{\mathcal{N}}(\check{\mathrm{z}})\right|, \\
& \left|\mathbb{I}_{\mathcal{M}}(\check{\mathrm{z}})-\mathbb{I}_{\mathcal{O}}(\check{\mathrm{z}})\right| \geq\left|\mathbb{I}_{\mathcal{N}}(\check{\mathrm{z}})-\mathbb{I}_{\mathcal{O}}(\check{\mathrm{z}})\right| ; \\
& \left|\mathbb{F}_{\mathcal{M}}(\check{\mathrm{z}})-\mathbb{F}_{\mathcal{O}}(\check{\mathrm{z}})\right| \geq\left|\mathbb{F}_{\mathcal{M}}(\check{\mathrm{z}})-\mathbb{F}_{\mathcal{N}}(\check{\mathrm{z}})\right|, \\
& \left|\mathbb{F}_{\mathcal{M}}(\check{\mathrm{z}})-\mathbb{F}_{\mathcal{O}}(\check{\mathrm{z}})\right| \geq\left|\mathbb{F}_{\mathcal{N}}(\check{\mathrm{z}})-\mathbb{F}_{\mathcal{O}}(\check{\mathrm{z}})\right| ; \\
& \text { Thus } \\
& \mathrm{F}_{S V N S}(\mathcal{M}, \mathcal{N}) \geq \mathrm{F}_{S V N S}(\mathcal{M}, \mathcal{O}) \\
& \text { and } \mathrm{F}_{S V N S}(\mathcal{N}, \mathcal{O}) \geq \mathrm{F}_{S V N S}(\mathcal{M}, \mathcal{O})
\end{aligned}
$$

Now TF is increasing in between $\left[0, \frac{\pi}{4}\right]$.

\section{DEPENDENCE OF SVN DECISION MAKING ON TANGENT SIMILARITY}

Suppose there exist a distinct set of students $\breve{\mathcal{A}}_{1}, \breve{\mathcal{A}}_{2}, \breve{\mathcal{A}}_{3}, \ldots, \breve{\mathcal{A}}_{\dot{m}}$ set of parameters of each student $\breve{\mathcal{C}}_{1}, \breve{\mathcal{C}}_{2}, \breve{\mathcal{C}}_{3}, \ldots, \breve{\mathcal{C}}_{\dot{n}}$, and the alternatives of each student $\widetilde{\mathcal{B}}_{1}, \breve{\mathcal{B}}_{2}, \breve{\mathcal{B}}_{3}, \ldots, \widetilde{\mathcal{B}}_{\dot{k}}$. The classification of alternatives corresponding to each student is given by decision maker. The classification gives the efficiency of students $\breve{\mathcal{A}}_{i}(i=$

\begin{tabular}{|c|c|c|c|c|}
\hline & $\overline{\mathcal{B}}_{\boldsymbol{k}}$ & $\breve{\mathcal{B}}_{\boldsymbol{k}}$ & $\ldots$ & $\widetilde{\mathcal{B}}_{\boldsymbol{k}}$ \\
\hline$\check{\boldsymbol{C}}_{1}$ & $\beta_{11}$ & $\beta_{12}$ & $\ldots$ & $\beta_{1 k}$ \\
\hline$\check{\boldsymbol{C}}_{2}$ & $\beta_{21}$ & $\beta_{22}$ & $\ldots$ & $\alpha_{2 \dot{k}}$ \\
\hline$\ldots$ & $\cdots$ & $\cdots$ & $\cdots$ & $\ldots$ \\
\hline$\check{\boldsymbol{C}}_{\dot{\boldsymbol{n}}}$ & $\beta_{\dot{n} 1}$ & $\beta_{\dot{n} 2}$ & $\ldots$ & $\beta_{\dot{n} k}$ \\
\hline
\end{tabular}
$1,2,3, \ldots, \dot{m})$ contrary to the parameter $\check{\mathcal{C}}_{1}(\mathrm{j}=1,2, \ldots, \dot{n})$.
For Multi Attribute Decision Making concern; values related to alternatives are given in the resulting decision matrix model as in Table (a) and Table (b). Relation among students and parameters can be represented in Table (a) and the relation among parameters and alternatives can be represented in Table (b)

Now $\alpha_{i \mathrm{j}}$ and $\beta_{i \mathrm{i}}$ all are SVNNs.

Here are phases for making decision.

PHASE 1: CALCULATION OF RELATION AMONG STUDENTS \& PARAMETERS

Relationship among students $\breve{\mathcal{A}}_{\boldsymbol{i}}(\boldsymbol{i}=\mathbf{1}, \mathbf{2}, \mathbf{3}, \ldots, \dot{\boldsymbol{m}})$ and parameters $\breve{\mathcal{C}}_{\mathfrak{j}}(\mathfrak{j}=\mathbf{1}, \mathbf{2}, \ldots, \dot{\boldsymbol{n}})$ is given by the table (c).

Table (c): In terms of single valued Neutrosophic sets the relation among students and parameters:

\begin{tabular}{c|c|c|c|c}
\hline \multicolumn{1}{c}{} & $\check{\mathcal{C}}_{1}$ & $\check{\mathcal{C}}_{2}$ & $\ldots$ & $\check{\mathcal{C}}_{\dot{n}}$ \\
\hline$\check{\mathcal{A}}_{1}$ & $\left(\mathbb{T}_{11}, \mathbb{I}_{11}, \mathbb{F}_{11}\right)$ & $\left(\mathbb{T}_{12}, \mathbb{I}_{12}, \mathbb{F}_{12}\right)$ & $\ldots$ & $\left(\mathbb{T}_{1 \dot{n}}, \mathbb{I}_{1 \dot{n}}, \mathbb{F}_{1 \dot{n}}\right)$ \\
$\check{\mathcal{A}}_{2}$ & $\left(\mathbb{T}_{21}, \mathbb{I}_{21}, \mathbb{F}_{21}\right)$ & $\left(\mathbb{T}_{22}, \mathbb{I}_{22}, \mathbb{F}_{22}\right)$ & $\ldots$ & $\left(\mathbb{T}_{2 \dot{n}}, \mathbb{I}_{2 \dot{n}}, \mathbb{F}_{2 \dot{n}}\right)$ \\
$\ldots$ & $\ldots$ & $\ldots$. & $\ldots$ & $\ldots$ \\
$\check{\mathcal{A}}_{\dot{m}}$ & $\left(\mathbb{T}_{\dot{m} 1}, \mathbb{I}_{\dot{m} 1}, \mathbb{F}_{\dot{m} 1}\right)$ & $\left(\mathbb{T}_{\dot{m} 2}, \mathbb{I}_{\dot{m} 2}, \mathbb{F}_{\dot{m} 2}\right)$ & $\ldots$ & $\left(\mathbb{T}_{\dot{m} \dot{n}}, \mathbb{I}_{\dot{m} \dot{n}}, \mathbb{F}_{\dot{m} \dot{m}}\right)$ \\
\hline
\end{tabular}

\begin{tabular}{|c|c|c|c|c|}
\hline & $\breve{\mathcal{B}}_{1}$ & $\breve{\mathcal{B}}_{2}$ & $\ldots$. & $\check{\mathcal{B}}_{\boldsymbol{k}}$ \\
\hline$\check{\mathcal{C}}_{1}$ & $\left(\mathbb{T}_{11}, \mathbb{I}_{11}, \mathbb{F}_{11}\right)$ & $\left(\mathbb{T}_{12}, \mathbb{I}_{12}, \mathbb{F}_{12}\right)$ & $\ldots$ & $\left(\mathbb{T}_{1 k}, \mathbb{I}_{1 k}, \mathbb{F}_{1 k}\right)$ \\
\hline$\check{\mathcal{C}}_{2}$ & $\left(\mathbb{T}_{21}, \mathbb{I}_{21}, \mathbb{F}_{21}\right)$ & $\left(\mathbb{T}_{22}, \mathbb{I}_{22}, \mathbb{F}_{22}\right)$ & $\ldots$ & $\left(\mathbb{T}_{2 k}, \mathbb{I}_{2 k}, \mathbb{F}_{2 k}\right)$ \\
\hline$\ldots$. & $\ldots$ & $\ldots$ & $\cdots$ & $\ldots$ \\
\hline$\check{\boldsymbol{C}}_{\dot{n}}$ & $\left(\mathbb{T}_{\mathfrak{n} 1}, \mathbb{I}_{\mathfrak{n} 1}, \mathbb{F}_{n 1}\right)$ & $\left(\mathbb{T}_{\mathfrak{n} 2}, \mathbb{I}_{\mathfrak{n} 2}, \mathbb{F}_{\mathfrak{n} 2}\right)$ & $\cdots$ & $\left(\mathbb{T}_{\pi k}, \mathbb{I}_{n h}, \mathbb{F}_{n k}\right)$ \\
\hline
\end{tabular}

PHASE 2: CALCULATION OF RELATION AMONG PARAMETERS \& ALTERNATIVES

Relation among parameters

$\check{\mathcal{C}}_{\mathfrak{j}}(\mathfrak{j}=1,2, \ldots, \dot{\boldsymbol{n}})$ And alternatives
$\breve{\mathcal{B}}_{\mathfrak{t}}(\mathfrak{t}=\mathbf{1}, \mathbf{2}, \ldots, \dot{\boldsymbol{k}})$ is given by the Table $(\mathrm{d})$.

Table (d): In terms of single valued Neutrosophic sets the relation among parameters and alternatives:
PHASE 3: CALCULATION OF RELATION AMONG PARAMETERS \& ALTERNATIVES

Similarity measure among the table (c) and table (d) is determined by using $\mathrm{T}_{\boldsymbol{S V N \boldsymbol { S }}}(\boldsymbol{M}, \mathcal{N})$.

\section{COSINE SIMILARITY MEASURE FOR SINGLE VALUED NEUTROSOPHIC SETS [43]}

\section{PHASE 4: RANKING THE ALTERNATIVES}

Maximum value reveals best Alternative.

PHASE 5: Stop.

Let $\check{X}=\left\{\check{z}_{1}, \breve{z}_{2}, \ldots, \breve{z}_{\dot{n}}\right\}$ and two single valued neutrosophic sets in $\check{X}$ are

$\left.\mathcal{M}=\left\{<\check{z}_{i}: \mathbb{T}_{\mathcal{M}}\left(\check{z}_{i}\right), \mathbb{I}_{\mathcal{M}}\left(\check{\mathrm{z}}_{i}\right), \mathbb{F}_{\mathcal{M}}\left(\check{\mathrm{z}}_{i}\right)\right\rangle, \check{\mathrm{z}}_{i} \in \check{X}\right\}$ and 


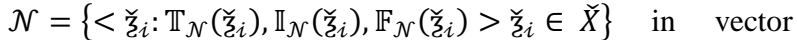
space, the cosine SM of single valued Neutrosophic sets can be expressed as

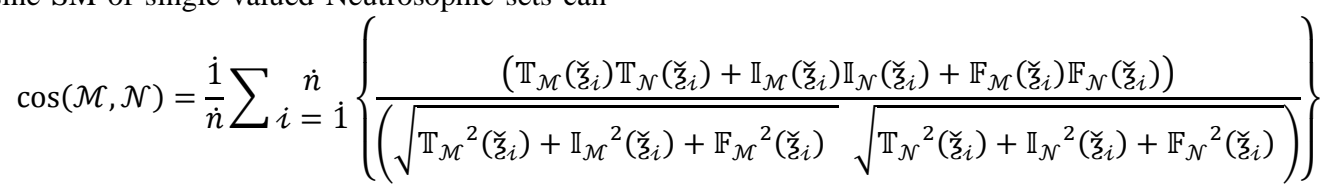

\section{COTANGENT SIMILARITY MEASURES FOR SINGLE VALUED NEUTROSOPHIC SETS [47]}

Let $\breve{X}=\left\{\breve{z}_{1}, \breve{z}_{2}, \ldots, \breve{z}_{\dot{n}}\right\}$ and two single valued neutrosophic sets in $\check{X}$ are $\mathcal{M}=\left\{<\check{z}_{i}: \mathbb{T}_{\mathcal{M}}\left(\check{z}_{i}\right), \mathbb{I}_{\mathcal{M}}\left(\check{z}_{i}\right), \mathbb{F}_{\mathcal{M}}\left(\check{z}_{i}\right)\right\rangle, \check{z}_{i} \in$ $\check{X}\}$ and $\mathcal{N}=\left\{<\check{z}_{i}: \mathbb{T}_{\mathcal{N}}\left(\check{z}_{i}\right), \mathbb{I}_{\mathcal{N}}\left(\check{z}_{i}\right), \mathbb{F}_{\mathcal{N}}\left(\check{z}_{i}\right)>\check{z}_{i} \in \check{X}\right\}$ the cotangent SM for above SVNSs can be expressed as:

$$
\cot (\mathcal{M}, \mathcal{N})=\frac{\dot{i}}{\dot{n}} \sum_{i=1}^{\dot{n}} \cot \left[\frac{\pi}{4}+\frac{\pi}{12}\left(\left|\mathbb{T}_{\mathcal{M}}\left(\check{z}_{i}\right)-\mathbb{T}_{\mathcal{N}}\left(\check{\mathfrak{z}}_{i}\right)\right|+\left|\mathbb{I}_{\mathcal{M}}\left(\check{z}_{i}\right)-\mathbb{I}_{\mathcal{N}}\left(\check{z}_{i}\right)\right|+\left|\mathbb{F}_{\mathcal{M}}\left(\check{\mathfrak{z}}_{i}\right)-\mathbb{F}_{\mathcal{N}}\left(\check{z}_{i}\right)\right|\right)\right]
$$

NOTE: The phases for determining the cosine and cotangent similarity measure is same as explained above in Section 4

\section{CASE STUDY: SELECTION OF AN ACADEMIC PROGRAMS FOR HIGHER SECONDARY SCHOOL}

Assume an effective instance that helps the candidates of secondary examination in selection of suitable subjects for higher secondary education. After matriculation, students put efforts in subjects of his choice to make bright his future .Mostly student get confused in selection of better career. They do not know which profession is suitable for them and sometimes take wrong decision that effects their future choosing a career which is inappropriate lead them to wrong path. The student must be attentive while choosing student according to his/ her taste so it is obligatory to follow the mathematical technique for making decision. The SM between candidate's vs parameters and parameters vs educational program. This technique include truthiness, indeterminacy and falseness function

Suppose there exist the set of candidates $\breve{\mathcal{A}}=\left\{\check{\mathcal{A}}_{1}, \breve{\mathcal{A}}_{2}, \check{\mathcal{A}}_{3}\right\}$, Set of educational program $\breve{\mathcal{B}}=\{$ arts and crafts $\left(\widetilde{\mathcal{B}}_{1}\right)$, accounting ( $\left.\widetilde{\mathcal{B}}_{2}\right)$, science $\left(\widetilde{\mathcal{B}}_{3}\right)$, professional series $\left.\left(\breve{\mathcal{B}}_{4}\right)\right\}$ and set of parameters $\check{\mathcal{C}}=\left\{\right.$ concentration $\left(\check{\mathcal{C}}_{1}\right)$, good grade point $\left(\check{\mathcal{C}}_{2}\right.$ ), interest in language $\left(\check{\mathcal{C}}_{3}\right)$,interest in mathematics $\left(\check{\mathcal{C}}_{4}\right)$, strenuous $\left.\left(\check{\mathcal{C}}_{5}\right)\right\}$.It is intended to examine candidates and help them to make decision for the selection of right educational program..

Phase 1:- In term of single valued Neutrosophic set, relation among candidates and parameters is given in Table (e). Table (e): Relation among candidates and parameters is:

\begin{tabular}{cccccc}
\hline $\mathbf{R}-1$ & $\check{\mathcal{C}}_{1}$ & $\check{\mathcal{C}}_{2}$ & $\check{\mathcal{C}}_{3}$ & $\check{\mathcal{C}}_{4}$ & $\check{\mathcal{C}}_{5}$ \\
\hline$\check{\mathcal{A}}_{1}$ & $\left(\begin{array}{l}0.8 \\
0.2 \\
0.2\end{array}\right)$ & $\left(\begin{array}{l}0.7 \\
0.2 \\
0.1\end{array}\right)$ & $\left(\begin{array}{l}0.6 \\
0.3 \\
0.3\end{array}\right)$ & $\left(\begin{array}{l}0.8 \\
0.3 \\
0.2\end{array}\right)$ & $\left(\begin{array}{l}0.5 \\
0.4 \\
0.2\end{array}\right)$ \\
\hline$\check{\mathcal{A}}_{2}$ & $\left(\begin{array}{l}0.7 \\
0.1 \\
0.3\end{array}\right)$ & $\left(\begin{array}{l}0.6 \\
0.3 \\
0.2\end{array}\right)$ & $\left(\begin{array}{l}0.7 \\
0.2 \\
0.1\end{array}\right)$ & $\left(\begin{array}{l}0.7 \\
0.4 \\
0.1\end{array}\right)$ & $\left(\begin{array}{l}0.8 \\
0.0 \\
0.2\end{array}\right)$ \\
\hline$\check{\mathcal{A}}_{3}$ & $\left(\begin{array}{l}0.5 \\
0.3 \\
0.3\end{array}\right)$ & $\left(\begin{array}{l}0.6 \\
0.3 \\
0.2\end{array}\right)$ & $\left(\begin{array}{l}0.7 \\
0.3 \\
0.1\end{array}\right)$ & $\left(\begin{array}{l}0.8 \\
0.0 \\
0.2\end{array}\right)$ & $\left(\begin{array}{l}0.8 \\
0.3 \\
0.1\end{array}\right)$
\end{tabular}

Phase 2: In term of single valued Neutrosophic set, relation among parameters and educational program is given in Table (f)

Table (f): Relation among parameters and academic programs is:

\begin{tabular}{ccccc}
\hline $\mathbf{R}-2$ & $\check{\mathcal{B}}_{1}$ & $\check{\mathcal{B}}_{2}$ & $\check{\mathcal{B}}_{3}$ & $\check{\mathcal{B}}_{4}$ \\
\hline$\check{\boldsymbol{C}}_{\mathbf{1}}$ & $\left(\begin{array}{l}0.8 \\
0.3 \\
0.1\end{array}\right)$ & $\left(\begin{array}{l}0.9 \\
0.1 \\
0.2\end{array}\right)$ & $\left(\begin{array}{l}0.7 \\
0.3 \\
0.2\end{array}\right)$ & $\left(\begin{array}{l}0.8 \\
0.2 \\
0.2\end{array}\right)$ \\
\hline$\check{\mathcal{C}}_{2}$ & $\left(\begin{array}{l}0.5 \\
0.4 \\
0.1\end{array}\right)$ & $\left(\begin{array}{l}0.6 \\
0.3 \\
0.2\end{array}\right)$ & $\left(\begin{array}{l}0.7 \\
0.4 \\
0.0\end{array}\right)$ & $\left(\begin{array}{l}0.9 \\
0.1 \\
0.1\end{array}\right)$ \\
\hline$\check{\mathcal{C}}_{3}$ & $\left(\begin{array}{l}0.6 \\
0.4 \\
0.1\end{array}\right)$ & $\left(\begin{array}{l}0.7 \\
0.3 \\
0.3\end{array}\right)$ & $\left(\begin{array}{l}0.7 \\
0.3 \\
0.2\end{array}\right)$ & $\left(\begin{array}{l}0.9 \\
0.1 \\
0.2\end{array}\right)$ \\
\hline
\end{tabular}




\begin{tabular}{|c|c|c|c|c|}
\hline$\check{\boldsymbol{C}}_{4}$ & $\left(\begin{array}{l}0.6 \\
0.2 \\
0.2\end{array}\right)$ & $\left(\begin{array}{l}0.7 \\
0.2 \\
0.2\end{array}\right)$ & $\left(\begin{array}{l}0.8 \\
0.2 \\
0.1\end{array}\right)$ & $\left(\begin{array}{l}0.5 \\
0.4 \\
0.2\end{array}\right)$ \\
\hline$\check{\mathcal{C}}_{5}$ & $\left(\begin{array}{l}0.7 \\
0.3 \\
0.2\end{array}\right)$ & $\left(\begin{array}{l}0.6 \\
0.3 \\
0.2\end{array}\right)$ & $\left(\begin{array}{l}0.7 \\
0.4 \\
0.1\end{array}\right)$ & $\left(\begin{array}{l}0.6 \\
0.4 \\
0.1\end{array}\right)$ \\
\hline
\end{tabular}

Phase 3:- Calculate the Tangent SM among the Table (e) and (f).

Table (g). Tangent similarity measure among Table (e) \& (f) is

\begin{tabular}{ccccc}
\hline TS Measures & $\check{\mathcal{B}}_{1}$ & $\breve{\mathcal{B}}_{2}$ & $\check{\mathcal{B}}_{3}$ & $\breve{\mathcal{B}}_{4}$ \\
\hline$\check{\mathcal{A}}_{1}$ & 0.92127 & $\mathbf{0 . 9 4 7 5 7}$ & 0.93707 & 0.92107 \\
\hline$\check{\mathcal{A}}_{2}$ & 0.90014 & $\mathbf{0 . 9 2 6 4 4}$ & 0.90533 & 0.87874 \\
\hline$\check{\mathcal{A}}_{3}$ & 0.92653 & 0.92096 & $\mathbf{0 . 9 3 1 7 7}$ & 0.86284 \\
\hline
\end{tabular}

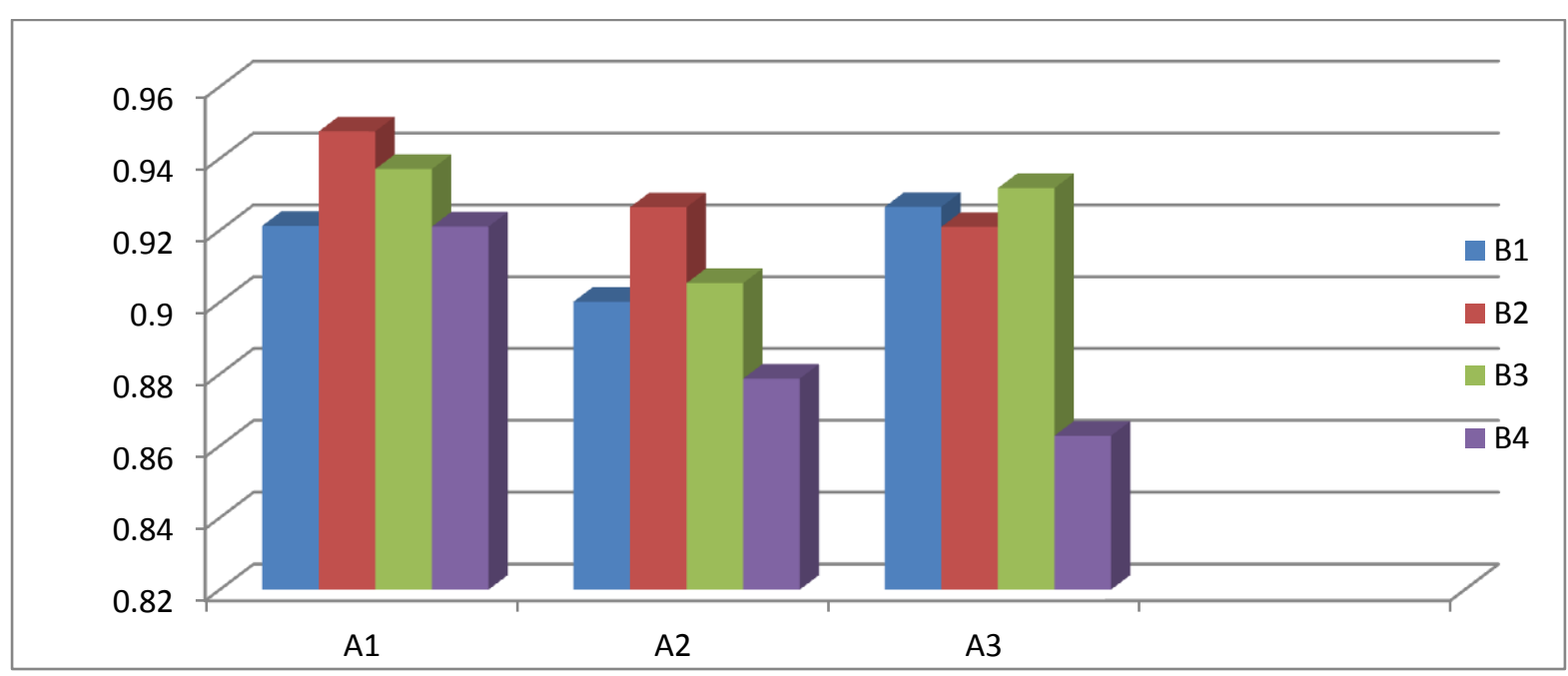

Phase 4:- The maximum values of similarity measure are 0.94757, 0.92644 and 0.93177 correspond to $\breve{\mathcal{A}}_{1}, \breve{\mathcal{A}}_{2}$ and $\check{\mathcal{A}}_{3}$. Here the maximum value will help students to make decision for the selection of academic programs.
Thus candidate $\breve{\mathcal{A}}_{1}$ should choose accounting program $\breve{\mathcal{A}}_{2}$ should choose accounting program and $\breve{\mathcal{A}}_{3}$ should choose science program.

Table (w). Cosine similarity measure among Table (e) \& (f) is

\begin{tabular}{ccccc}
\hline Cosine Measures & $\check{\mathcal{B}}_{1}$ & $\check{\mathcal{B}}_{2}$ & $\check{\mathcal{B}}_{3}$ & $\check{\mathcal{B}}_{4}$ \\
& & & & \\
\hline$\check{\mathcal{A}}_{1}$ & 0.96512 & $\mathbf{0 . 9 8 7 3 2}$ & 0.97906 & 0.96891 \\
\hline$\check{\mathcal{A}}_{2}$ & 0.95011 & $\mathbf{0 . 9 6 1 9 4}$ & 0.94597 & 0.93913 \\
\hline$\check{\mathcal{A}}_{3}$ & $\mathbf{1 . 0 1 0 5 5}$ & 0.96034 & 0.97362 & 0.91675 \\
\hline
\end{tabular}




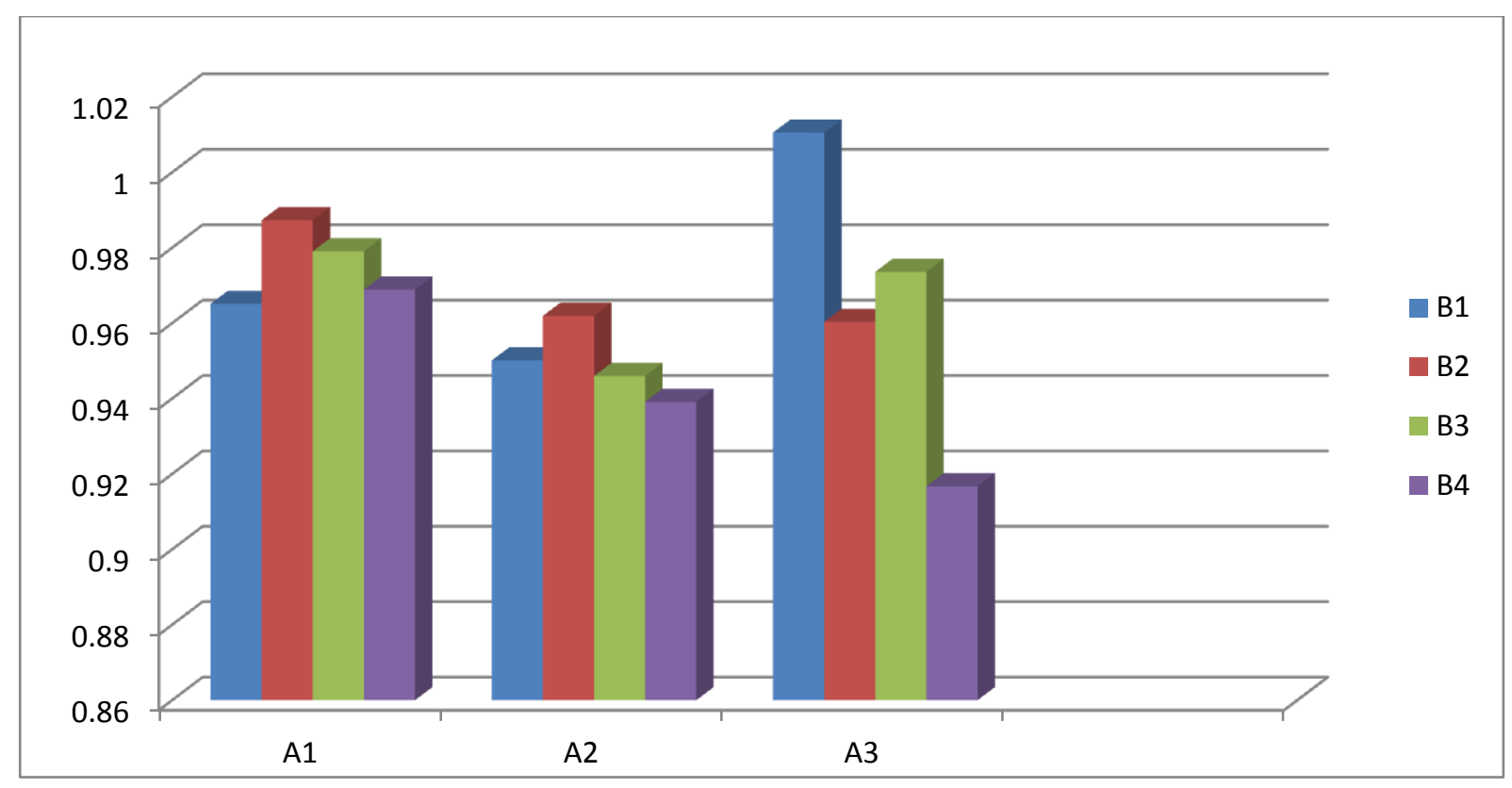

Candidate $\breve{\mathcal{A}}_{1}$ should choose accounting program $\breve{\mathcal{A}}_{2}$ should choose accounting program and $\check{\mathcal{A}}_{3}$ should choose arts and crafts.

Table (x). Cotangent similarity measure among Table (e) \& (f) is

\begin{tabular}{ccccc}
\hline $\begin{array}{c}\text { Cotangent } \\
\text { Measures }\end{array}$ & $\breve{\mathcal{B}}_{1}$ & $\breve{\mathcal{B}}_{2}$ & $\breve{\mathcal{B}}_{3}$ & $\breve{\mathcal{B}}_{4}$ \\
\hline$\check{\mathcal{A}}_{1}$ & 0.8759 & $\mathbf{0 . 9 0 0 8 2}$ & 0.8818 & 0.85812 \\
\hline$\check{\mathcal{A}}_{2}$ & 0.8189 & $\mathbf{0 . 8 6 5 8}$ & 0.8289 & 0.7862 \\
\hline$\check{\mathcal{A}}_{3}$ & 0.8382 & $\mathbf{0 . 8 6 6 9}$ & 0.8645 & 0.7685 \\
\hline
\end{tabular}

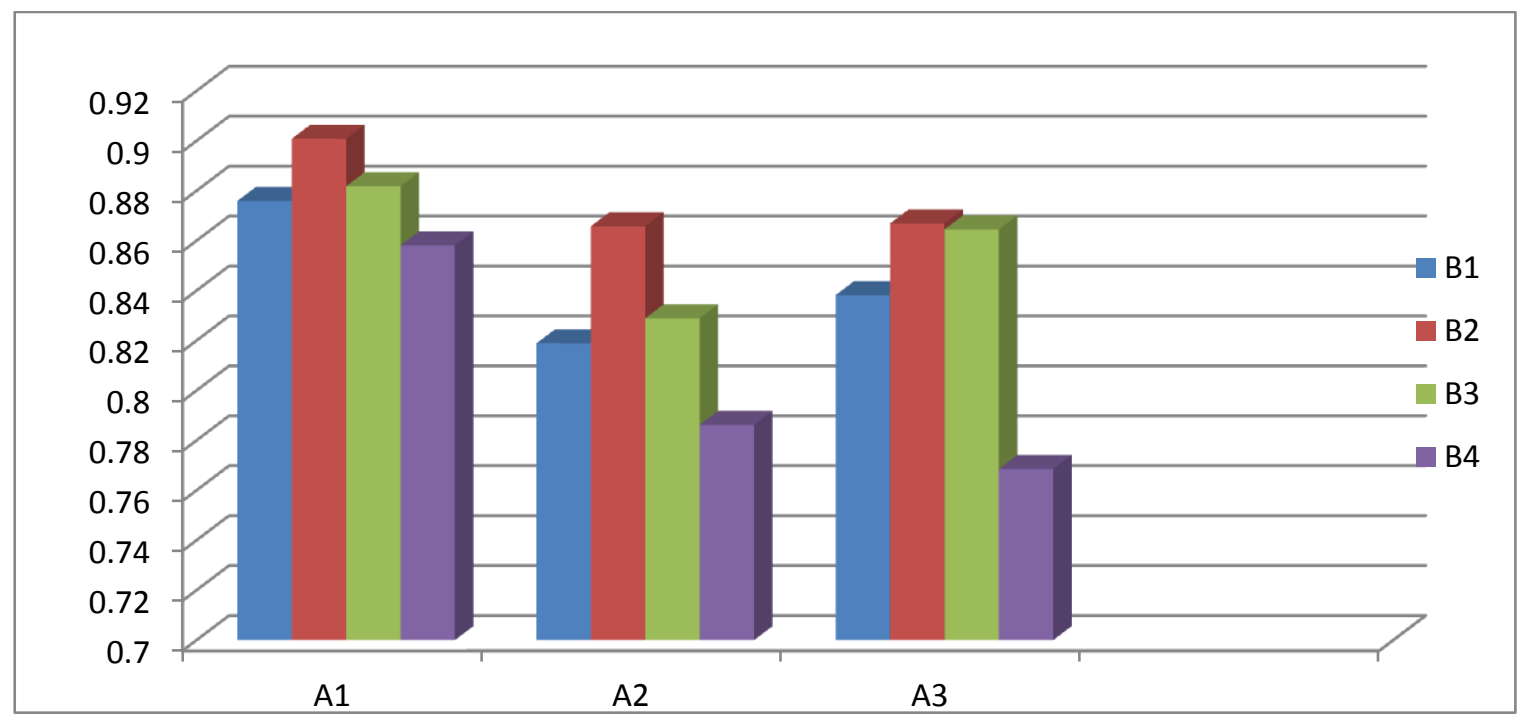

Candidate $\breve{\mathcal{A}}_{1}$ should choose accounting program, $\breve{\mathcal{A}}_{2}$ and $\breve{\mathcal{A}}_{3}$ should also choose accounting.

\section{CONCLUSION}

This article described tangent, cosine, cotangent similarity measure for single valued Neutrosophic sets and its applications in realistic problem of selection of an academic programs. In this article we have observed that $\widetilde{\mathcal{B}}_{2}$ educational program is the best for the candidates $\breve{\mathcal{A}}_{1}$ and $\check{\mathcal{A}}_{2}$ and $\breve{\mathcal{B}}_{1}$ is best for $\breve{\mathcal{A}}_{3}$. According to this study it is observed that cosine similarity gives values very near to 1 means $100 \%$ similarity. So this study gives the result that the cosine Measure is the best and easy to solve and gives more accurate results then other measures. 


\section{REFERENCES}

[1] Ansari.Q.A, Biswas.R and Aggarwal.S, (2011).Proposal for applicability of neutrosophic set theory medical AI, International Journal of computer Applications,27(5):511

[2] Atanassov.K, (1986).Intuitionistic fuzzy sets, Fuzzy sets and systems, 20:87-96.

[3] Atanassov.K, Gargov.G, (1989). Interval valued Intuitionistic fuzzy sets, Fuzzy sets and system, 31:343349.

[4] Biswas.P, Pramanik.S, and Giri.C.B, (2015). Cosine similarity measure based multi-attribute decision making with trapezoidal fuzzy neutrosophic numbers, Neutrosophic Sets and System, 8: 47-58.

[5] Broumi.S, and, Smarandache.F, (2013). Several similarity measures of neutrosophic sets, Neutrosophic Sets and Systems 1: 54-62.

[6] Broumi.S, and Smarandache.F, (2013). Correlation coefficient of interval neutrosophic set, Periodical of Applied Mechanics and Materials, with the title Engineering Decisions and Scientific Research in Aerospace, Robotics, Biomechanics, Mechanical Engineering and Manufacturing, Proceedings of the International Conference ICMERA, Bu-charest, 436.

[7] Broumi.S and Samarandache.F, (2014).Cosine similarity measure of interval valued neutrosophic set,Neutrosophic sets and systems, 5:15-20.

[8] Cheng.D.H and Guo.Y, (2008).A new neutrosophic approach to image thresholding, New mathematics and Natural computation, 4(3):291-308

[9] Chen.M.S,Yeh.M.S and Hsiao.H.P, (1995).A comparison of Similarity measure of fuzzy value, fuzzy sets and system,72:79-89.

[10] Chen.M.S, (1998).A new approach to handling fuzzy decision making problems, IEEE Transactions on systems man and Cybemetics, 8: 1012-1016.

[11] Guo.Y and Cheng.D.H,(2009).New neutrosophic approach to image segmentation, Pattern recognition, 42: $587-595$

[12] Hyung.K.L, Song.S.Y and Lee.M.K, (1994).Similarity measure between fuzzy sets and between elements, Fuzzy sets and systems, 62:291-293.

[13] Jafar.N.M, Saqlain.M, Saeed.M, Abbas.Q (2020), Application of Soft-Set Relations and Soft Matrices in Medical Diagnosis using Sanchez's Approach , International Journal of Computer Applications, 177(32): 7-11.

[14] Jafar.N.M, Muniba.K, Saeed.A, Abbas.S, Bibi.I (2019), Application of Sanchez's Approach to Disease Identification Using Trapezoidal Fuzzy Numbers, International Journal of Latest Engineering Research and Applications, 4(9):51-57

[15] Jafar.N.M, Faizullah, Shabbir.S, Alvi.F.M.S, Shaheen.L (2020), Intuitionistic Fuzzy Soft Matrices, Compliments and Their Relations with Comprehensive Study of Medical Diagnosis, International Journal of Latest Engineering Research and Applications, 5(1): 23-30.

[16] Jafar.N.M, Saeed.A, Waheed.M, Shafiq.A (2020), A
Comprehensive Study of Intuitionistic Fuzzy Soft Matrices and its Applications in Selection of Laptop by Using Score Function, International Journal of Computer Applications, 177(38): 8-17.

[17] Jafar.N.M， Khan.R.M， , Sultan.H，Ahmad.N (2020), Interval Valued Fuzzy Soft Sets and Algorithm of IVFSS Applied to the Risk Analysis of Prostate Cancer, International Journal of Computer Applications, 177(38): $18-26$

[18] Jafar.N.M， Imran.R， Hassan.S， Riffat.A， Shuaib.R (2020), Medical Diagnosis Using Neutrosophic Soft Matrices and Their Compliments, International Journal of Advanced Research in Computer Science, 11(1):1-3

[19] Kharal.A, (2013). A neutrosophic multi-criteria decision making method, New Mathematics and natural computation Creighton University USA.

[20] Majumder,P and Samanta.K.S, (2014). On similarity and entropy of neutrosophic sets, Journal of Intelligent and Fuzzy Systems 26:1245-1252

[21] Mondal.K and Pramanik.S, (2014).Multi-criteria group decision making approach for teacher recruitment in higher education under simplified neutrosophic environment, Neutrosophic sets and system, 6:28-34.

[22] Mondal .K and Pramanik.S, (2015).Neutrosophic decision making model of school choice, Neutrosophic sets and systems, 7:62-68.

[23] Mondal.K and Paramanik.S, (2014).A study on problems of Hijras in west Bengal based on neutrosophic cognitive maps, Neutrospohic sets and system, 5:21-26.

[24] Mondal.K and Pramanik.S, (2015).Intuitimistic fuzzy similarity measure based on tangent function and its application to multi attribute decision, Global Journal of Advanced Research,2(2):404-471.

[25] Pappis.C.P and Karacapilidis .I.N, (1993).A comparative assessment of measure of similarity of fuzzy values, Fuzzy sets and system, 56:171-174

[26] Pramanik.S and Chackrabarti.N.S,(2013).A study on problems of construction workers in west Bengal based on neutrosophic cognitive maps, International Journal of innovative Research in science, engineering and technology,2(11),6387-6394.

[27] Pramanik.S, Roy.K.T, (2014).Neutrosophcic game theoretic approach to Indo-Pak conflict over JammuKashmir, neutrosophic sets and systems, 2:82-101.

[28] Riaz.M, Saeed.M, Saqlain.M, Jafar.N (2019), Impact of Water Hardness in Instinctive Laundry System Based on Fuzzy Logic Controller, Punjab University Journal of Mathematics, 51(4):73-84

[29] Saeed, M., Zulqarnain, M. and Dayan, F. (2018). TOPSIS analysis for the prediction of diabetes based on general characteristics of humans. Int. J. of Pharm. Sci. and Research. 9: 2932-2939

[30] Saqlain.M.Jafar.N, Riffat.A (2018), Smart Phone Selection by Consumers' In Pakistan: FMCGDM Fuzzy Multiple Criteria Group Decision Making Approach, Gomal University Journal of Research, 34 (1): 27-31.

[31] Saqlain.M, Jafar.N, Hamid.R,Shahzad.A. (2019), Prediction of Cricket World Cup 2019 by TOPSIS 
Technique of MCDM-A Mathematical Analysis, International Journal of Scientific \& Engineering Research, 10(2): 789-792

[32] Saqlain.M, Naz.K, Ghaffar.K, Jafar.N.M (2019), Fuzzy Logic Controller: The Impact of Water $\mathrm{pH}$ on Detergents, Scientific Inquiry of Review 3(3):16-29

[33] Saqlain M, Saeed M, Ahmad M. R, Smarandache F, (2019), Generalization of TOPSIS for Neutrosophic Hypersoft set using Accuracy Function and its Application, Neutrosophic Sets and Systems (NSS), 27: 131-137.

[34] Smarandache.F, (1998). A unifying field in logics, neutrosophy: neutrosophic probability set and logic, Rehoboth American Res.press: 1-141

[35] Smarandache.F,(1999).Linguistic paradoxes and tautologies, Libertas Mathematica,Universityof Texas at Arlington IX :143-154.

[36] Szmidt.E and Kacprzyk.J, (2004).Medical diagnostic reasoning using a similarity measure for intuitionistic fuzzy sets, Notes on intuitionistic fuzzy sets, 10(4):61-69.

[37] Tian.MY, (3012).A new fuzzy similarity based on cotangent function for medical diagnosis, Adv. Model option, 5(2):151-156.

[38] Wang.H, Smarandache.F, Zhang.Y and Sunderraman.R, (2010).Single valued Neutrosophic sets, Multi space and Multistructure, 4:410-413.

[39] Wang.J.W,(1997).New similarity measure on fuzzy sets and element, fuzzy sets and system,85:305-309.

[40] Ye.J,(2013).Multicriteria decision making method using the correlation coefficient using single valued neutrosophic environment, International Journal of general system, $\mathbf{4 2 ( 4 ) : 3 8 6 - 3 9 4 . ~}$

[41] Ye.J, and Zhang.Q,(2012). Single valued neutrosophic similarity measures for multiple attribute decision making Neutrosophic Sets and System 2: 48-54.

[42] Ye.J, (2014).Similarity measures between interval neutrosophic sets and their multi criteria decision making method, Journal of Intelligent and Fuzzy Systems 26:165-172

[43] Ye.J, (2014).Vectors similarity measure of simplified neutrosophic sets and their application in multi criteria decision making, International Journal of fuzzy systems, 16(2):204-215

[44] Ye.J, (2014) Improved cosine similarity measures of simplified neutrosophic sets for medical diagnoses, Artificial Intelligence in Medicine doi: 10.1016/j.artmed.2014.12.007.

[45] Ye.S and Ye.J ,(2014).Dice similarity measure between single valued neutrosophic multi sets and its application in medical diagnosis, Neurosophic sets and systems, 6:49-54.

[46] Ye.S,Fu.J and Ye.J ,(2014).Medical diagnosis sing distance based similarity measures for single valued neutrosophic multi sets, Neutrosophic sets and system, $7: 47-52$

[47] Ye.J,(2015).Single valued neutrosophic similarity measures based on cotangent function and their application in the fault diagnosis of steam turbine,Soft comput,DOI 10.1007/s00500-015-1818-y.

[48] Zadeh.A.L, (1965).Fuzzy sets, Information and control, 8:338-353.

[49] Zadeh.A.L, (1975).The concept of linguistic variable and its application to approximate reasoning I , Inform sci, 8:199-249.

[50] Zhang.M,Zhang.L and Cheng.D.H,(2010).A neutrosophic approach to image segmentation based on watershed method, single processing,90(5):1510-1517. 